\title{
Existence and multiplicity of solutions of second-order discrete Neumann problem with singular $\phi$-Laplacian operator
}

Yanqiong Lu* and Ruyun Ma

\section{"Correspondence:}

linmu8610@163.com

Department of Mathematics,

Northwest Normal University,

Lanzhou, 730070, P.R. China

\begin{abstract}
In this paper, we obtain the existence and multiplicity of solutions for discrete Neumann boundary value problem with singular $\phi$-Laplacian operator $\nabla\left(\frac{\Delta u_{k}}{\sqrt{1-\kappa\left(\Delta u_{k}\right)^{2}}}\right)+r_{k} u_{k}+f\left(k, u_{k}, \Delta u_{k}\right)=0,2 \leq k \leq N-1, \Delta u_{1}=0=\Delta u_{N-1}$ by using upper and lower solutions method and Brouwer degree theory, where $\kappa>0$ is a constant, $\mathbf{r}=\left(r_{2}, \ldots, r_{N-1}\right) \in \mathbb{R}^{N-2}$, and $f$ is a continuous function. We also give some examples to illustrate the main results.
\end{abstract}

MSC: 34B10; 34B18

Keywords: $\operatorname{singular} \phi$-Laplacian; existence; Neumann problem; Brouwer degree; upper and lower solutions

\section{Introduction}

In this paper we present some existence and multiplicity results for the discrete Neumann boundary value problem with singular $\phi$-Laplacian operator

$$
\begin{aligned}
& \nabla\left(\frac{\Delta u_{k}}{\sqrt{1-\kappa\left(\Delta u_{k}\right)^{2}}}\right)+r_{k} u_{k}+f\left(k, u_{k}, \Delta u_{k}\right)=0, \quad k \in[2, N-1]_{\mathbb{Z}} \\
& \Delta u_{1}=0=\Delta u_{N-1}
\end{aligned}
$$

where $\kappa>0$ is a constant, $\Delta$ is the forward difference operator defined by $\Delta u_{k}=u_{k+1}-u_{k}$, $\nabla$ is the backward difference operator defined by $\nabla u_{k}=u_{k}-u_{k-1}, \mathbf{r}=\left(r_{2}, \ldots, r_{N-1}\right) \in \mathbb{R}^{N-2}$, $f:[2, N-1]_{\mathbb{Z}} \times \mathbb{R}^{2} \rightarrow \mathbb{R}$ is a continuous function and $[2, N-1]_{\mathbb{Z}}:=\{2,3, \ldots, N-1\}$ with $N \geq 4$ is an integer.

This problem originated from the study of hypersurfaces in the Lorentz-Minkowski space with coordinates $\left(x_{1}, \ldots, x_{N}, t\right)$ and the metric $\sum_{j=1}^{N}\left(d x_{j}\right)^{2}-(d t)^{2}$ leads to partial differential equations (PDE) of the type

$$
\operatorname{div}\left(\frac{\nabla v(x)}{\sqrt{1-|\nabla v(x)|^{2}}}\right)=H(x, v(x)) \text { in } \Omega
$$

where $\Omega$ is a domain in $\mathbb{R}^{N}(N \geq 1)$ and $H: \Omega \times \mathbb{R} \rightarrow \mathbb{R}$ is a nonlinearity prescribing the mean curvature of the hypersurface. A first essential result concerning the above PDE was

\section{Springer}

( 2014 Lu and Ma; licensee Springer. This is an Open Access article distributed under the terms of the Creative Commons Attribution License (http://creativecommons.org/licenses/by/2.0), which permits unrestricted use, distribution, and reproduction in any medium, provided the original work is properly cited. 
proved by Calabi [1] in the case $\Omega=\mathbb{R}^{N}$ and $N \leq 4$. This was later extended to arbitrary dimension by Cheng and Yau in [2]. On the other hand, if $H \equiv c>0$ and $\Omega=\mathbb{R}^{N}$, then Treibergs [3] obtained an existence result about entire solutions for (1.2) in the presence of a pair of well-ordered upper and lower solutions, and (1.2) coupled with the Neumann boundary conditions has been considered by López [4] and Bereanu et al. [5-7]. For existence and multiplicity results concerning (positive) solutions of the classical case $(\kappa=0)$, see for example $[8,9]$, and for other results concerning the Neumann boundary value problems, see [10] and their references.

This paper addresses a question of interest regarding the discrete Neumann problem (1.1):

Under what conditions does the discrete Neumann problem (1.1) have at least one solution?

Particular significance in the above question lies in the fact that strange and interesting distinctions can occur between the theory of differential equations and the theory of difference equations. For example, properties such as existence, uniqueness, and multiplicity of solutions may not be shared between the theory of differential equations and the theory of difference equations $[11,12]$, even though the right-hand side of the equations under consideration may be the same. Moreover, when investigating difference equations, as opposed to differential equations, basic ideas from calculus are not necessarily available, such as the intermediate value theorem, the mean value theorem, and the Rolle theorem. Thus, one faces new challenges and innovation is required.

It is worth to point out that corresponding results for the discrete Neumann problem (1.1) with $r_{k} \equiv r \geq 0$ and $\kappa=1$ have been proved in [13,14]. The classical case has been studied by $[15,16]$. It is interesting to remark that, in contrast to the classical case, the discrete Neumann problem with relativistic acceleration

$$
\nabla\left(\frac{\Delta u_{k}}{\sqrt{1-\left(\Delta u_{k}\right)^{2}}}\right)+r u_{k}=e_{k}, \quad k \in[2, N-1]_{\mathbb{Z}}, \quad \Delta u_{1}=0=\Delta u_{N-1}
$$

has at least one solution for any $r \neq 0$ and any forcing term $\mathbf{e}$ (see [14, Corollary 2 and Remark 2]).

In order to explain the main result, let us introduce some notation. For any $x \in \mathbb{R}$, we write $x^{+}=\max \{x, 0\}$ and $x^{-}=\max \{-x, 0\}$. For $\mathbf{e}=\left(e_{2}, \ldots, e_{N-1}\right) \in \mathbb{R}^{N-2}$, we put $E=\sum_{k=2}^{N-1} e_{k}$, $E_{ \pm}=\sum_{k=2}^{N-1} e_{k}^{ \pm}, \overline{\mathbf{e}}=\frac{1}{N-2} \sum_{k=2}^{N-1} e_{k}$ and note that $E=E_{+}-E_{-}$.

Motivated by the above results from [13-18], we consider the discrete Neumann problem (1.1) under the nonlinearity satisfying some suitable conditions and obtain the existence and multiplicity of solutions of (1.1). We shall show that if $\overline{\mathbf{r}} \neq 0$ and $f$ is bounded, then (1.1) has at least one solution; see Theorem 3.1. Moreover, suppose that $f \operatorname{does}$ not depend on $\Delta u_{k}$ in (1.1) and $\overline{\mathbf{r}}>0$, then (1.1) has at least one solution if either $f$ is superlinear at zero and sublinear at infinity (Corollary 3.1) or $f$ is sublinear at zero and superlinear at infinity and $\mathbf{r}>0$ (Corollary 3.2).

On the other hand, Bereanu and Mawhin [14] dealt with the Ambrosetti-Prodi type results for the problem (1.1) with $\mathbf{r}=0, f\left(k, u_{k}, \Delta u_{k}\right)=g\left(k, u_{k}, \Delta u_{k}\right)-s$, they obtain the result that there exists $s_{0} \in \mathbb{R}\left(s_{1} \in \mathbb{R}\right)$ such that problem (1.1) has zero, at least one or at least two solutions according to $s<s_{0}, s=s_{0}$ or $s>s_{0}\left(s>s_{1}, s=s_{1}\right.$ or $\left.s<s_{1}\right)$ if $g\left(k, u_{k}, \Delta u_{k}\right) \rightarrow+\infty$ $\left(g\left(k, u_{k}, \Delta u_{k}\right) \rightarrow-\infty\right)$, as $\left|u_{k}\right| \rightarrow \infty$ uniformly for $\Delta u_{k} \in\left(-\frac{1}{\sqrt{\kappa}}, \frac{1}{\sqrt{\kappa}}\right)$; see [14, Theorem 6, 
Theorem 7 and Remark 9]. We note that these results also hold for the problem (1.1) by the same argument in [14, Theorem 6, Theorem 7]. Naturally we can ask: what would happen if $f$ is null at infinity? Theorem 3.4 will give the existence, multiplicity, and nonexistence of solutions of (1.1) when $f$ is null at infinity.

The rest of the paper is organized as follows. In Section 2, we introduce some notations, auxiliary results and present the method of lower and upper solutions. In addition, we also introduce the method to construct lower and upper solutions. In Section 3 we give some applications to deal with the discrete Neumann problem with various nonlinearities such as the nonlinearity is bounded and super-sub linear perturbations, the nonlinearity is null at infinity and the nonlinearity is singular. We also give some examples to illustrate the main results.

\section{Some notations and the method of lower and upper solutions}

In the sequel, let us introduce some notations. Let $a, b \in \mathbb{N}$ with $a<b$, we denote $[a, b]_{\mathbb{Z}}:=$ $\{a, a+1, \ldots, b\}$. In addition, we denote $\sum_{s=a}^{b} u_{s}=0$ with $b<a$ and $\prod_{s=a}^{b} u_{s}=1$ with $b<a$.

For $\mathbf{u}=\left(u_{1}, \ldots, u_{p}\right) \in \mathbb{R}^{p}$, set $\|\mathbf{u}\|_{\infty}=\max _{1 \leq k \leq p}\left|u_{k}\right|,\|\mathbf{u}\|_{1}=\sum_{k=1}^{p}\left|u_{k}\right|$. If $\boldsymbol{\alpha}, \boldsymbol{\beta} \in \mathbb{R}^{p}$, we write $\boldsymbol{\alpha} \leq \boldsymbol{\beta}$ (resp. $\boldsymbol{\alpha}<\boldsymbol{\beta}$ ) if $\alpha_{k} \leq \beta_{k}$ (resp. $\alpha_{k}<\beta_{k}$ ) for all $1 \leq k \leq p$. The following assumption upon $\phi$ (called singular) is made throughout the paper:

$\left(H_{\phi}\right) \phi:(-a, a) \rightarrow \mathbb{R}(0<a<\infty)$ is an increasing homeomorphism with $\phi(0)=0$.

The model example is

$$
\phi(s)=\frac{s}{\sqrt{1-\kappa s^{2}}}, \quad s \in\left(-\frac{1}{\sqrt{\kappa}}, \frac{1}{\sqrt{\kappa}}\right) .
$$

Let $N \in \mathbb{N}$ with $N \geq 4$ be fixed and $\mathbf{u}=\left(u_{1}, u_{2}, \ldots, u_{N}\right) \in \mathbb{R}^{N}$. Then we denote

$$
\Delta \mathbf{u}=\left(\Delta u_{1}, \ldots, \Delta u_{N-1}\right) \in \mathbb{R}^{N-1}
$$

by $\Delta u_{k}=u_{k+1}-u_{k}$ for $k \in[1, N-1]_{\mathbb{Z}}$ and if $\|\Delta \mathbf{u}\|_{\infty}:=\max _{k \in[1, N-1]_{\mathbb{Z}}}\left|\Delta u_{k}\right|<a$, define

$$
\nabla[\phi(\Delta \mathbf{u})]=\left(\nabla\left[\phi\left(\Delta u_{2}\right)\right], \ldots, \nabla\left[\phi\left(\Delta u_{N-1}\right)\right]\right) \in \mathbb{R}^{N-2}
$$

by $\nabla\left[\phi\left(\Delta u_{k}\right)\right]=\phi\left(\Delta u_{k}\right)-\phi\left(\Delta u_{k-1}\right)$ for $k \in[2, N-1]_{\mathbb{Z}}$.

Let $f:[2, N-1]_{\mathbb{Z}} \times \mathbb{R}^{2} \rightarrow \mathbb{R}$ be a continuous function. Then its Nemytskii operator $N_{f}(\mathbf{u}): \mathbb{R}^{N} \rightarrow \mathbb{R}^{N-2}$ is given by

$$
N_{f}(\mathbf{u})=\left(f\left(2, u_{2}, \Delta u_{2}\right), \ldots, f\left(N-1, u_{N-1}, \Delta u_{N-1}\right)\right) .
$$

It follows that $N_{f}$ is continuous and takes bounded sets into bounded sets.

Let $P, Q$ be the projectors defined by

$$
P \mathbf{u}=u_{2}, \quad \overline{\mathbf{u}}=Q \mathbf{u}=\frac{1}{N-2} \sum_{k=2}^{N-1} u_{k} \quad \text { for all } \mathbf{u} \in \mathbb{R}^{N}
$$


If $\mathbf{u} \in \mathbb{R}^{N}$, we write $\tilde{\mathbf{u}}=\mathbf{u}-\overline{\mathbf{u}}$ and we shall consider the following closed subspaces of $\mathbb{R}^{N}$ :

$$
\begin{aligned}
& W^{N-2}=\left\{\mathbf{u} \in \mathbb{R}^{N} \mid \Delta u_{1}=0=\Delta u_{N-1}\right\}, \\
& \tilde{W}^{N-2}=\left\{\mathbf{u} \in W^{N-2} \mid \overline{\mathbf{u}}=0\right\} .
\end{aligned}
$$

Let the vector space $W^{N-2}$ be endowed with the orientation of $\mathbb{R}^{N}$ and the norm $\|\mathbf{u}\|_{\infty}=\max _{1 \leq k \leq N}\left|u_{k}\right|$. Its elements can be associated to the coordinates $\left(u_{2}, \ldots, u_{N-1}\right)$ and correspond to the elements of $\mathbb{R}^{N}$ of the form

$$
\left(u_{2}, u_{2}, u_{3}, \ldots, u_{N-1}, u_{N-1}\right)
$$

For $\mathbf{u}^{0} \in W^{N-2}$, we set $B\left(\mathbf{u}^{0}, \rho\right):=\left\{\mathbf{u} \in W^{N-2} \mid\|\mathbf{u}\|_{\infty}<\rho\right\}(\rho>0)$ and, for brevity, we shall write $B_{\rho}$ instead of $B(\mathbf{0}, \rho)$.

Now, we recall the following technical result given as Proposition 4 and Proposition 6 in $[14]$.

Lemma 2.1 Let $F: \mathbb{R}^{N} \rightarrow \mathbb{R}^{N-2}$ be a continuous operator which takes bounded sets into bounded sets and consider the abstract discrete Neumann problem

$$
\nabla(\phi(\Delta \mathbf{u}))=F(\mathbf{u}), \quad \Delta u_{1}=0=\Delta u_{N-1}
$$

A function $\mathbf{u}$ is a solution of (2.1) if and only if $\mathbf{u} \in W^{N-2}$ is a fixed point of the continuous operator $\mathcal{A}_{F}: W^{N-2} \rightarrow W^{N-2}$ defined by $\mathcal{A}_{F}(\mathbf{u})=\mathbf{v}$, where $\mathbf{v}=\left(v_{1}, v_{2}, \ldots, v_{N}\right) \in W^{N-2}$ satisfying

$$
v_{2}=u_{2}+Q N_{F}(\mathbf{u}), \quad v_{k}=u_{2}+Q N_{F}(\mathbf{u})+\sum_{j=2}^{k-1} \phi^{-1}\left(\sum_{l=2}^{j} F\left(u_{k}\right)\right), \quad k \in[3, N-1]_{\mathbb{Z}} .
$$

Furthermore, $\|\Delta(\mathcal{A}(\mathbf{u}))\|_{\infty}<$ a for all $\mathbf{u} \in W^{N-2}$ and

$$
\|\tilde{\mathbf{u}}\|_{\infty}<a(N-2)
$$

for any solution $\mathbf{u}$ of (2.1).

Let us consider the discrete Neumann problem

$$
\nabla\left[\phi\left(\Delta u_{k}\right)\right]=f\left(k, u_{k}, \Delta u_{k}\right), \quad k \in[2, N-1]_{\mathbb{Z}}, \quad \Delta u_{1}=0=\Delta u_{N-1} .
$$

Obviously, from Lemma 2.1, the fixed point operator associated to (2.3) is

$$
\mathcal{A}_{f}(\mathbf{u})=\mathbf{u} \text {. }
$$

In what follows, we present the method of lower and upper solutions for difference equations (see [14, Theorem 3]) to the Neumann boundary value problem (2.3). 
Definition 2.1 A function $\boldsymbol{\alpha}=\left(\alpha_{1}, \ldots, \alpha_{N}\right)$ (resp. $\left.\boldsymbol{\beta}=\left(\beta_{1}, \ldots, \beta_{N}\right)\right)$ is called a lower solution (resp. an upper solution) for (2.3) if $\|\Delta \alpha\|_{\infty}<a$ (resp. $\|\Delta \beta\|_{\infty}<a$ ) and

$$
\begin{aligned}
& \nabla\left[\phi\left(\Delta \alpha_{k}\right)\right] \geq f\left(k, \alpha_{k}, \Delta \alpha_{k}\right) \\
& \text { (resp. } \left.\nabla\left[\phi\left(\Delta \beta_{k}\right)\right] \leq f\left(k, \beta_{k}, \Delta \beta_{k}\right)\right), \quad k \in[2, N-1]_{\mathbb{Z}}, \\
& \Delta \alpha_{1} \geq 0, \quad \Delta \alpha_{N-1} \leq 0 \quad\left(\text { resp. } \Delta \beta_{1} \leq 0, \Delta \beta_{N-1} \geq 0\right) .
\end{aligned}
$$

Such a lower or an upper solution is called strict if the inequality $(2.4)$ is strict.

We need the following result, which can be proved by the strategy of the proof of Theorem 3 in [14]; see [14, Remark 8].

Lemma 2.2 If (2.3) has a lower solution $\boldsymbol{\alpha}=\left(\alpha_{1}, \alpha_{2}, \ldots, \alpha_{N}\right)$ and an upper solution $\boldsymbol{\beta}=$ $\left(\beta_{1}, \beta_{2}, \ldots, \beta_{N}\right)$ such that $\boldsymbol{\alpha} \leq \boldsymbol{\beta}$, then (2.3) has a solution $\mathbf{u}$ such that $\boldsymbol{\alpha} \leq \mathbf{u} \leq \boldsymbol{\beta}$. Moreover, if $\boldsymbol{\alpha}$ and $\boldsymbol{\beta}$ are strict, then $\boldsymbol{\alpha}<\mathbf{u}<\boldsymbol{\beta}$, and

$$
\operatorname{deg}\left[I-\mathcal{A}_{f}, \Omega_{\boldsymbol{\alpha}, \boldsymbol{\beta}}, \mathbf{0}\right]=-1,
$$

where $\Omega_{\alpha, \beta}=\left\{\mathbf{u} \in W^{N-2} \mid \boldsymbol{\alpha}<\mathbf{u}<\boldsymbol{\beta},\|\Delta \mathbf{u}\|_{\infty}<a\right\}$.

Notice that Lemma 2.2 proved that the problem (2.3) has at least one solution if it has a lower solution $\alpha$ and an upper solution $\beta$ with $\alpha \leq \beta$. In the following result we prove some additional results concerning the location of the solution. In particular we have $a$ posteriori estimations which will be very useful in the sequel (Remark 2.1).

Theorem 2.1 Assume that (2.3) has a lower solution $\boldsymbol{\alpha}$ and an upper solution $\boldsymbol{\beta}$ such that

$$
\exists k_{\star} \in[1, N]_{\mathbb{Z}}: \alpha_{k_{\star}}>\beta_{k_{\star}} .
$$

Then (2.3) has at least one solution $\mathbf{u}$ such that

$$
\min \left\{\alpha_{k_{u}}, \beta_{k_{u}}\right\} \leq u_{k_{u}} \leq \max \left\{\alpha_{k_{u}}, \beta_{k_{u}}\right\} \text { for some } k_{u} \in[1, N]_{\mathbb{Z}}
$$

Proof Let

$$
\begin{aligned}
& u^{*}=\|\alpha\|_{\infty}+\|\beta\|_{\infty}+a(N-2), \\
& m=\max \left\{|f(k, u, v)|+1 \mid(k, u, v) \in[2, N-1]_{\mathbb{Z}} \times\left[-u^{*}-2, u^{*}+2\right] \times[-a, a]\right\},
\end{aligned}
$$

and define the continuous function $g:[2, N-1]_{\mathbb{Z}} \times \mathbb{R}^{2} \rightarrow \mathbb{R}$ by

$$
g(k, u, v)= \begin{cases}-m-1, & u \leq-u^{*}-1, \\ f(k, u, v)+\left(u+u^{*}\right)(m+1+f(k, u, v)), & -u^{*}-1<u<-u^{*}, \\ f(k, u, v), & -u^{*} \leq u \leq u^{*}, \\ f(k, u, v)+\left(u-u^{*}\right) m, & u^{*}<u<u^{*}+1, \\ f(k, u, v)+m, & u \geq u^{*}+1 .\end{cases}
$$


Let us consider the modified Neumann problem

$$
\nabla\left[\phi\left(\Delta u_{k}\right)\right]=g\left(k, u_{k}, \Delta u_{k}\right), \quad \Delta u_{1}=0=\Delta u_{N-1},
$$

and let $\mathcal{A}_{g}$ be the fixed point operator associated to (2.8).

It is not difficult to verify that $\alpha$ is a lower solution and $\beta$ is an upper solution of the problem (2.8). Moreover, by computation, $\alpha_{1}=-u^{*}-2$ is a lower solution of (2.8) and $\beta_{1}=u^{*}+2$ is an upper solution of $(2.8)$. Notice that

$$
\alpha_{1}<\min \{\alpha, \beta\} \leq \max \{\alpha, \beta\}<\beta_{1},
$$

which, together with (2.6), implies that

$$
\Omega_{\alpha_{1}, \beta} \cup \Omega_{\alpha, \beta_{1}} \subset \Omega_{\alpha_{1}, \beta_{1}}, \quad \Omega_{\alpha_{1}, \beta} \cap \Omega_{\alpha, \beta_{1}}=\emptyset .
$$

So, we can consider the open bounded set

$$
\Omega=\Omega_{\alpha_{1}, \beta_{1}} \backslash\left[\overline{\Omega_{\alpha_{1}, \beta}} \cup \overline{\Omega_{\alpha, \beta_{1}}}\right] .
$$

It follows that

$$
\Omega=\left\{u \in \Omega_{\alpha_{1}, \beta_{1}} \mid u_{k_{u}}>\beta_{k_{u}}, u_{s_{u}}<\alpha_{s_{u}} \text { for some } k_{u}, s_{u} \in[1, N]_{\mathbb{Z}}\right\}
$$

and

$$
\partial \Omega=\partial \Omega_{\alpha_{1}, \beta_{1}} \cup \partial \Omega_{\alpha_{1}, \beta} \cup \partial \Omega_{\alpha, \beta_{1}} .
$$

Clearly, any constant function between $\beta_{k_{\star}}$ and $\alpha_{k_{\star}}$ is contained in $\Omega$, so $\Omega \neq \emptyset$.

Next, let us consider $\mathbf{u} \in \partial \Omega$ such that $\mathcal{A}_{f}(\mathbf{u})=\mathbf{u}$ and $\|\mathbf{u}\|_{\infty}=u^{*}+2$. Notice that one has $\|\Delta \mathbf{u}\|_{\infty}<a$. This implies that there exists $k_{0} \in[2, N-1]_{\mathbb{Z}}$ such that $u_{k_{0}}=\max _{k \in[1, N]_{\mathbb{Z}}} u_{k}=$ $u^{*}+2$ or $u_{k_{0}}=\min _{k \in[1, N]_{\mathbb{Z}}} u_{k}=-u^{*}-2$. In the first case we can assume that $k_{0} \in[2, N-$ $1]_{\mathbb{Z}}$, then $\Delta u_{k_{0}} \leq 0, \Delta u_{k_{0}-1} \geq 0$. This, together with $\phi$ is an increasing homeomorphism, implies $\nabla\left[\phi\left(\Delta u_{k_{0}}\right)\right] \leq 0$. On the other hand, we have

$$
\nabla\left[\phi\left(\Delta u_{k_{0}}\right)\right]=f\left(k_{0}, u_{k_{0}}, \Delta u_{k_{0}}\right)+m>0,
$$

which is a contradiction. Analogously, one can obtain a contradiction in the second case. Consequently,

$$
\left[\mathbf{u} \in \partial \Omega, \mathcal{A}_{g}(\mathbf{u})=\mathbf{u}\right] \quad \Rightarrow \quad\|\mathbf{u}\|_{\infty}<u^{*}+2
$$

Now, let $\mathbf{u} \in \partial \Omega$ be such that $\mathcal{A}_{g}(\mathbf{u})=\mathbf{u}$. It follows from (2.9) that $\|\mathbf{u}\|_{\infty}<u^{*}+2,\|\Delta \mathbf{u}\|_{\infty}<$ $a$ and $\mathbf{u} \in \partial \Omega_{\alpha_{1}, \beta} \cup \partial \Omega_{\alpha, \beta_{1}}$. We infer that there exists $k_{0} \in[1, N]_{\mathbb{Z}}$ such that $u_{k_{0}}=\alpha_{k_{0}}$ or $u_{k_{0}}=\beta_{k_{0}}$, implying that $\left|u_{k_{0}}\right| \leq\|\alpha\|_{\infty}+\|\beta\|_{\infty}$. Then

$$
\left|u_{k}\right| \leq\left|u_{k_{0}}\right|+\sum_{s=2}^{N-1}\left|\Delta u_{s}\right|<u^{*} \quad \text { for all } k \in[1, N]_{\mathbb{Z}},
$$


and, consequently,

$$
\left[\mathbf{u} \in \partial \Omega, \mathcal{A}_{g}(\mathbf{u})=\mathbf{u}\right] \quad \Rightarrow \quad\|\mathbf{u}\|_{\infty}<u^{*}
$$

We have distinguished two cases to discuss.

Case 1. Assume that there exists $\mathbf{u} \in \partial \Omega$ such that $\mathcal{A}_{g}(\mathbf{u})=\mathbf{u}$. Using (2.10), we deduce that $\|\mathbf{u}\|_{\infty}<u^{*}$, implying that $\mathbf{u}$ is a solution of (2.3) and (2.7) holds. Actually, in this case there exists $k_{u} \in[1, N]_{\mathbb{Z}}$ such that $u_{k_{u}}=\alpha_{k_{u}}$ or $u_{k_{u}}=\beta_{k_{u}}$.

Case 2. Assume that $\mathcal{A}_{g}(\mathbf{u}) \neq \mathbf{u}$ for all $\mathbf{u} \in \partial \Omega$. Then, from Lemma 2.2 applied to $g$, it follows that

$$
\operatorname{deg}\left[I-\mathcal{A}_{g}, \Omega_{\alpha_{1}, \beta_{1}}, \mathbf{0}\right]=\operatorname{deg}\left[I-\mathcal{A}_{g}, \Omega_{\alpha_{1}, \beta}, \mathbf{0}\right]=\operatorname{deg}\left[I-\mathcal{A}_{g}, \Omega_{\alpha, \beta_{1}}, \mathbf{0}\right]=-1
$$

This, together with the additivity property of the Brouwer degree, implies that

$$
\operatorname{deg}\left[I-\mathcal{A}_{g}, \Omega, \mathbf{0}\right]=1
$$

which, together with the existence property of the Brouwer degree, implies that there exists $\mathbf{u} \in \Omega$ such that $\mathcal{A}_{g}(\mathbf{u})=\mathbf{u}$. It follows that there exist $k_{1}, k_{2} \in[1, N]_{\mathbb{Z}}$ such that $u_{k_{1}}<\alpha_{k_{1}}$ and $u_{k_{2}}>\beta_{k_{2}}$. Then, using once again that $\|\Delta \mathbf{u}\|_{\infty}<a$, it follows that $\|\mathbf{u}\|_{\infty}<u^{*}$, and $\mathbf{u}$ is a solution of (2.3). Moreover, from $\mathbf{u} \in \Omega$ it follows that (2.7) is true.

Remark 2.1 Assume that (2.3) has a lower solution $\alpha$ and an upper solution $\beta$. From Lemma 2.2 and Theorem 2.1, we deduce that (2.3) has at least one solution $\mathbf{u}$ satisfying (2.7). In particular,

$$
\|\mathbf{u}\|_{\infty}<\|\alpha\|_{\infty}+\|\beta\|_{\infty}+a(N-2) .
$$

Remark 2.2 The corresponding result for second-order continuous periodic problems has been proved in Theorem 1 of [17] by the proof using the same strategy as above.

The following result is a particular case of [14, Lemma 6 and Remark 8] for the discrete Neumann boundary value problem.

Lemma 2.3 Let $\tilde{\mathbf{u}}=\left(\tilde{u}_{1}, \tilde{u}_{2}, \ldots, \tilde{u}_{N}\right) \in \tilde{W}^{N-2}$. Then the discrete Neumann problem

$$
\begin{aligned}
& \nabla\left[\phi\left(\Delta \tilde{u}_{k}\right)\right]=f\left(k, l+\tilde{u}_{k}, \Delta \tilde{u}_{k}\right)-\frac{1}{N-2} \sum_{j=2}^{N-1} f\left(k, l+\tilde{u}_{j}, \Delta \tilde{u}_{k}\right), \quad k \in[2, N-1]_{\mathbb{Z}}, \\
& \Delta u_{1}=0=\Delta u_{N-1}
\end{aligned}
$$

has at least one solution for all $l \in \mathbb{R}$.

The next result is an elementary estimation of the function $u \in W^{N-2}$.

Lemma 2.4 Let $u \in W^{N-2}$. Then

$$
\max _{k \in[1, N]_{\mathbb{Z}}} u_{k}-\min _{k \in[1, N]_{\mathbb{Z}}} u_{k} \leq(N-2)\|\Delta \mathbf{u}\|_{\infty}
$$


Proof Let $k_{*} \in[2, N-1]_{\mathbb{Z}}$ be such that $u_{k_{*}}=\min _{k \in[1, N]_{\mathbb{Z}}} u_{k}$ and $k^{*} \in[2, N-1]_{\mathbb{Z}}$ be such that $u_{k^{*}}=\max _{k \in[1, N]_{\mathbb{Z}}} u_{k}$. If $k^{*}=k_{*}$, then $u_{k^{*}}-u_{k_{*}}=0 \leq(N-2)\|\Delta \mathbf{u}\|_{\infty}$. If $k^{*}>k_{*}$, then

$$
u_{k^{*}}-u_{k_{*}}=\sum_{s=k_{*}}^{k^{*}-1} \Delta u_{s} \leq(N-2)\|\Delta \mathbf{u}\|_{\infty} .
$$

If $k^{*}<k_{*}$, then

$$
u_{k^{*}}-u_{k_{*}}=\sum_{s=k^{*}}^{k_{*}-1}\left(-\Delta u_{s}\right) \leq(N-2)\|\Delta \mathbf{u}\|_{\infty} .
$$

Therefore, it follows that

$$
u_{k^{*}}-u_{k_{*}} \leq(N-2)\|\Delta \mathbf{u}\|_{\infty}
$$

and the proof is completed.

In the following, we give a method to construct the lower solution and upper solution of the discrete Neumann problem

$$
\nabla\left[\phi\left(\Delta u_{k}\right)\right]=g_{0}\left(k, u_{k}\right)+e_{k}, \quad k \in[2, N-1]_{\mathbb{Z}}, \quad \Delta u_{1}=0=\Delta u_{N-1},
$$

where $g_{0}:[2, N-1]_{\mathbb{Z}} \times(0, \infty) \rightarrow \mathbb{R}$ is a continuous singular nonlinearity and $\mathbf{e}=$ $\left(e_{2}, \ldots, e_{N-1}\right) \in \mathbb{R}^{N-2}$.

The following result gives a method to construct a lower solution to (2.14), getting also control over its localization.

Theorem 2.2 Suppose that there exist $u^{1}>0$ and $\mathbf{c}=\left(c_{2}, \ldots, c_{N-1}\right) \in \mathbb{R}^{N-2}$ such that

$$
g_{0}(k, u) \leq c_{k}, \quad \forall(k, u) \in[2, N-1]_{\mathbb{Z}} \times\left[u^{1}, u^{1}+a(N-2)\right] .
$$

If

$$
\overline{\mathbf{c}}+\overline{\mathbf{e}} \leq 0,
$$

then (2.14) has a lower solution $\alpha$ such that

$$
u^{1} \leq \alpha<u^{1}+a(N-2)
$$

Proof Consider the function $\boldsymbol{\psi}=\mathbf{c}+\mathbf{e}$. We have two cases.

Case 1. Assume that $\Psi_{+}=0$. Taking $\alpha \equiv u^{1}$ and, using $\mathbf{c}+\mathbf{e} \leq 0$, it follows from (2.15) that $\alpha$ is a lower solution of (2.14).

Case 2. Assume that $\Psi_{+}>0$. Let $h_{k}=\psi_{k}^{+} \Psi_{-}-\psi_{k}^{-} \Psi_{+}$. Then using

$$
\sum_{k=2}^{N-1} h_{k}=\sum_{k=2}^{N-1}\left[\psi_{k}^{+} \Psi_{-}-\psi_{k}^{-} \Psi_{+}\right]=0
$$


and [14, Proposition 6], it follows that there exists $\mathbf{w} \in W^{N-2}$ such that

$$
\nabla\left[\phi\left(\Delta w_{k}\right)\right]=h_{k}, \quad \Delta w_{1}=0=\Delta w_{N-1}
$$

Let us take $u^{0}=1 / \Psi_{+}$and $\varpi_{j}=\min \left\{0, \phi^{-1}\left(\sum_{l=2}^{j} u^{0} h_{l}\right)\right\}$ for $j=2, \ldots, N-1$. Then we define

$$
\begin{aligned}
& \alpha_{2}=u^{1}-\min _{k \in[3, N-1]_{\mathbb{Z}}} \sum_{j=2}^{k-1} \varpi_{j}, \\
& \alpha_{k}=u^{1}+\sum_{j=2}^{k-1} \phi^{-1}\left(\sum_{l=2}^{j} u^{0} h_{l}\right)-\min _{k \in[3, N-1]_{\mathbb{Z}}} \sum_{j=2}^{k-1} \varpi_{j}, \quad k \in[3, N-1]_{\mathbb{Z}} .
\end{aligned}
$$

Let $\alpha_{1}=\alpha_{2}, \alpha_{N}=\alpha_{N-1}$, then $\Delta \alpha_{1}=0=\Delta \alpha_{N-1}$. On the other hand, we have

$$
\Delta \alpha_{k}=\phi^{-1}\left(\sum_{l=2}^{k} u^{0} h_{l}\right), \quad 2 \leq k \leq N-1
$$

Since $\min _{k \in[3, N-1]_{\mathbb{Z}}} \sum_{j=2}^{k-1} \varpi_{j} \leq 0$, Lemma 2.4 implies (2.17). Now, using (2.16), it follows that $\Psi_{+} \leq \Psi_{-}$, implying that

$$
\nabla\left[\phi\left(\Delta \alpha_{k}\right)\right]=u^{0} h_{k}=u_{0}\left[\psi_{k}^{+} \Psi_{-}-\psi_{k}^{-} \Psi_{+}\right] \geq \psi_{k}, \quad k \in[2, N-1]_{\mathbb{Z}}
$$

From (2.15) and (2.17), we deduce that

$$
g_{0}\left(k, \alpha_{k}\right)+e_{k} \leq \psi_{k}, \quad \forall k \in[2, N-1]_{\mathbb{Z}}
$$

Consequently,

$$
\nabla\left[\phi\left(\Delta \alpha_{k}\right)\right] \geq g_{0}\left(k, \alpha_{k}\right)+e_{k}, \quad \forall k \in[2, N-1]_{\mathbb{Z}}
$$

By a similar argument, it is easy to prove the following theorem.

Theorem 2.3 Suppose that there exist $u^{2}>0$ and $\mathbf{d}=\left(d_{2}, \ldots, d_{N-1}\right) \in \mathbb{R}^{N-2}$ such that

$$
g_{0}(k, u) \geq d_{k}, \quad \text { for any }(k, u) \in[2, N-1]_{\mathbb{Z}} \times\left[u^{2}, u^{2}+a(N-2)\right]
$$

If

$$
\overline{\mathbf{d}}+\overline{\mathbf{e}} \geq 0
$$

then (2.14) has an upper solution $\beta$ such that

$$
u^{2} \leq \beta<u^{2}+a(N-2) .
$$




\section{Some applications}

\subsection{Bounded and super-sub linear perturbations}

In this section we will study the discrete Neumann problem

$$
\nabla\left[\phi\left(\Delta u_{k}\right)\right]+r_{k} u_{k}=f\left(k, u_{k}, \Delta u_{k}\right), \quad \Delta u_{1}=0=\Delta u_{N-1}
$$

where $\mathbf{r}=\left(r_{2}, \ldots, r_{N-1}\right) \in \mathbb{R}^{N-2}$ and $f:[2, N-1]_{\mathbb{Z}} \times \mathbb{R}^{2} \rightarrow \mathbb{R}$ is a continuous function.

In the following theorem we prove that if $\overline{\mathbf{r}} \neq 0$ and $f$ is bounded on $[2, N-1]_{\mathbb{Z}} \times \mathbb{R} \times$ $(-a, a)$, then (3.1) has at least one solution. So, resonance occurs only when $\overline{\mathbf{r}}=0$.

Theorem 3.1 If $\overline{\mathbf{r}} \neq 0$ and $f$ is bounded on $[2, N-1]_{\mathbb{Z}} \times \mathbb{R} \times(-a, a)$, then (3.1) has at least one solution.

Proof Let $p>0$ be a constant such that

$$
|f(k, u, v)| \leq p \quad \text { for all }(k, u, v) \in[2, N-1]_{\mathbb{Z}} \times \mathbb{R} \times(-a, a) .
$$

For any $\lambda \in[0,1]$, let us consider the discrete Neumann problem

$$
\begin{aligned}
& \nabla\left[\phi\left(\Delta u_{k}\right)\right]=\lambda\left[N_{f}(\mathbf{u})-r_{k} u_{k}\right]+(1-\lambda)\left[Q N_{f}(\mathbf{u})-Q(\mathbf{r u})\right] \\
& \Delta u_{1}=0=\Delta u_{N-1} .
\end{aligned}
$$

Let $\mathcal{A}(\lambda, \cdot): W^{N-2} \rightarrow W^{N-2}$ be the fixed point operator associated to (3.2) by Lemma 2.1. Notice that if $\mathbf{u} \in W^{N-2}$ is such that $\mathbf{u}=\mathcal{A}(\lambda, \mathbf{u})$, then (3.2) is satisfied and

$$
Q N_{f}(\mathbf{u})=Q(\mathbf{r u}),
$$

implying that

$$
\overline{\mathbf{u}}=\frac{1}{\overline{\mathbf{r}}} Q\left[N_{f}(\mathbf{u})-\mathbf{r} \tilde{\mathbf{u}}\right] .
$$

So, one has

$$
|\overline{\mathbf{u}}|<\frac{p+a(N-2)\|\mathbf{r}\|_{\infty}}{\overline{\mathbf{r}}} .
$$

Then, for any $\rho>0$ sufficiently large, one has

$$
\mathbf{u} \neq \mathcal{A}(\lambda, \mathbf{u}) \quad \text { for all }(\lambda, \mathbf{u}) \in[0,1] \times \partial B_{\rho} .
$$

The invariance under homotopy of the Brouwer degree implies that

$$
\operatorname{deg}\left(I-\mathcal{A}(0, \cdot), B_{\rho}, \mathbf{0}\right)=\operatorname{deg}\left(I-\mathcal{A}(1, \cdot), B_{\rho}, \mathbf{0}\right) .
$$

Notice that from $Q^{2}=Q$ it follows that

$$
\mathcal{A}(0, \mathbf{u})=P \mathbf{u}+Q\left[N_{f}(\mathbf{u})-\mathbf{r u}\right], \quad \mathbf{u} \in W^{N-2} .
$$


So, the range of the operator $\mathcal{A}(\lambda, \cdot)$ is contained in the space of constant functions which is isomorphic to $\mathbb{R}$. Hence, using the reduction property of the Brouwer degree we deduce that, for $\rho$ sufficiently large,

$$
\operatorname{deg}\left(I-\mathcal{A}(0, \cdot), B_{\rho}, \mathbf{0}\right)=\operatorname{deg}\left(I-\left.\mathcal{A}(0, \cdot)\right|_{\mathbb{R}},(-\rho, \rho), 0\right)
$$

which, together with the fact that $f$ is bounded and

$$
\left[I-\left.\mathcal{A}(0, \cdot)\right|_{\mathbb{R}}\right](u)=\overline{\mathbf{r}} u-\frac{1}{N-2} \sum_{k=2}^{N-1} f(k, u, 0), \quad u \in \mathbb{R},
$$

implies that

$$
\operatorname{deg}\left(I-\mathcal{A}(0, \cdot), B_{\rho}, \mathbf{0}\right)=\operatorname{sign} \overline{\mathbf{r}} .
$$

We infer that

$$
\operatorname{deg}\left(I-\mathcal{A}(1, \cdot), B_{\rho}, \mathbf{0}\right) \neq 0
$$

and the existence property of the Brouwer degree implies that $\mathcal{A}(1, \cdot)$ has at least one fixed point $\mathbf{u}$ which is also a solution of (3.1).

Example 3.1 Consider the discrete Neumann problem with attractive singularity

$$
\begin{aligned}
& \nabla\left(\frac{\Delta u_{k}}{\sqrt{1-\kappa\left(\Delta u_{k}\right)^{2}}}\right)+g\left(\Delta u_{k}\right)+r_{k} u_{k}=e_{k}, \quad k \in[2, N-1]_{\mathbb{Z}}, \\
& \Delta u_{1}=0=\Delta u_{N-1},
\end{aligned}
$$

where $\kappa>0$ is a constant, $\mathbf{r}=\left(r_{2}, \ldots, r_{N-1}\right), \mathbf{e}=\left(e_{2}, \ldots, e_{N-1}\right) \in \mathbb{R}^{N-2}$ and $g: \mathbb{R} \rightarrow \mathbb{R}$ is a continuous function. If $\overline{\mathbf{r}} \neq 0$, then the above problem has at least one solution. In fact, let $\phi(s)=\frac{s}{\sqrt{1-\kappa s^{2}}}, f\left(k, u_{k}, \Delta u_{k}\right)=e_{k}-g\left(\Delta u_{k}\right)$. Then

$$
\left|f\left(k, u_{k}, \Delta u_{k}\right)\right| \leq\|\mathbf{e}\|_{\infty}+\max _{\Delta u_{k} \in\left[-\frac{1}{\sqrt{k}}, \frac{1}{\sqrt{k}}\right]} g\left(\Delta u_{k}\right)=: \rho .
$$

So, the result follows from Theorem 3.1.

In the following theorem we assume that $f$ is superlinear at zero and sublinear at infinity and we prove that (3.1) has at least one nontrivial solution if $\overline{\mathbf{r}}>0$.

Theorem 3.2 Assume that $f$ does not depends on $\Delta u_{k}$ in (3.1). If one has $\overline{\mathbf{r}}>0$ and

$$
\liminf _{u \rightarrow 0^{+}} \frac{f(k, u)}{u}>\max _{k \in[2, N-1]_{\mathbb{Z}}} r_{k} \geq \overline{\mathbf{r}}>\limsup _{u \rightarrow+\infty} \frac{f(k, u)}{u} \quad \text { uniformly for } k \in[2, N-1]_{\mathbb{Z}}
$$

then (3.1) has at least one nontrivial solution. 
Proof First of all, our assumption implies that there exists $\beta>0$ such that

$$
r_{k} \beta \leq f(k, \beta) \quad \text { for all } k \in[2, N-1]_{\mathbb{Z}} .
$$

This means that $\beta$ is an upper solution of (3.1).

On the other hand, from (3.4), there exist $\varepsilon>0$ and $u^{1}>\max \left\{\frac{a(N-2) \overline{\mathbf{r}^{+}}}{\varepsilon}, \beta\right\}$ such that

$$
f\left(k, u_{k}\right) \leq(\overline{\mathbf{r}}-\varepsilon) u_{k}, \quad k \in[2, N-1]_{\mathbb{Z}}, u_{k} \geq u^{1} .
$$

We will apply Theorem 2.2 with $g_{0}\left(k, u_{k}\right)=f\left(k, u_{k}\right)-r_{k} u_{k}$ and

$$
c_{k}=-r_{k} u^{1}+a(N-2) r_{k}^{-}+\max _{u_{k} \in\left[u^{1}, u^{1}+a(N-2)\right]} f\left(k, u_{k}\right), \quad \forall k \in[2, N-1]_{\mathbb{Z}} .
$$

Notice that

$$
\begin{aligned}
-r_{k} u_{k} & =r_{k}^{-} u_{k}-r_{k}^{+} u_{k} \leq r_{k}^{-}\left(u^{1}+a(N-2)\right)-r_{k}^{+} u^{1} \\
& =-r_{k} u^{1}+a(N-2) r_{k}^{-}, \quad \forall\left(k, u_{k}\right) \in[2, N-1]_{\mathbb{Z}} \times\left[u^{1}, u^{1}+a(N-2)\right],
\end{aligned}
$$

implying that (2.15) holds. Next, we have

$$
\overline{\mathbf{c}} \leq-u^{1} \overline{\mathbf{r}}+a(N-2) \overline{\mathbf{r}^{-}}+(\overline{\mathbf{r}}-\varepsilon)\left(u^{1}+a(N-2)\right) \leq 0 .
$$

Hence, from Theorem 2.2 we deduce that (3.1) has a lower solution $\alpha$ such that $u^{1} \leq \alpha<$ $u^{1}+a(N-2)$. In particular $\beta \leq \alpha$, and using Theorem 2.1, we infer that (3.1) has at least one solution $\mathbf{u}$ such that $\beta \leq u_{k_{u}}$, for some $k_{u} \in[1, N]_{\mathbb{Z}}$, which is also a nontrivial solution.

\section{Corollary 3.1 If $\overline{\mathbf{r}}>0$ and}

$$
\lim _{u \rightarrow 0_{+}} \frac{f(k, u)}{u}=\infty, \quad \lim _{u \rightarrow+\infty} \frac{f(k, u)}{u}=0, \quad \text { uniformly for } k \in[2, N-1]_{\mathbb{Z}}
$$

then (3.1) has at least one nontrivial solution.

Example 3.2 Consider the discrete Neumann problem with attractive singularity

$$
\nabla\left(\frac{\Delta u_{k}}{\sqrt{1-\kappa\left(\Delta u_{k}\right)^{2}}}\right)+r_{k} u_{k}=\left|u_{k}\right|^{\lambda}, \quad k \in[2, N-1]_{\mathbb{Z}}, \quad \Delta u_{1}=0=\Delta u_{N-1},
$$

where $\kappa>0$ is a constant, $\mathbf{r}=\left(r_{2}, \ldots, r_{N-1}\right) \in \mathbb{R}^{N-2}$ and $\lambda>0$. If $\overline{\mathbf{r}}>0$ and $\lambda \in(0,1)$, then the above problem has at least one solution.

The following dual result also holds, that is, $f$ is superlinear at infinity and sublinear at zero and we prove that (3.1) has at least one nontrivial solution if $\mathbf{r}>0$. 
Theorem 3.3 Assume that $f$ does not depend on $\Delta u_{k}$ in (3.1). If one has $\overline{\mathbf{r}}>0$ and

$$
\liminf _{u \rightarrow+\infty} \frac{f(k, u)}{u}>\overline{\mathbf{r}} \geq \min _{k \in[2, N-1]_{\mathbb{Z}}} r_{k}>\limsup _{u \rightarrow 0^{+}} \frac{f(k, u)}{u} \quad \text { uniformly for } k \in[2, N-1]_{\mathbb{Z}}
$$

then (3.1) has at least one nontrivial solution.

Proof Obviously, the assumption (3.5) implies that there exists $\alpha>0$ such that

$$
f(k, \alpha) \leq r_{k} \alpha \quad \text { for all } k \in[2, N-1]_{\mathbb{Z}} .
$$

This means that $\alpha$ is an upper solution of (3.1).

On the other hand, it follows from (3.5) that there exist $\varepsilon>0$ and $u^{2}>\max \left\{\frac{a(N-2) \overline{\mathbf{r}^{+}}}{\varepsilon}, \alpha\right\}$ such that

$$
f\left(k, u_{k}\right) \geq(\overline{\mathbf{r}}+\varepsilon) u_{k}, \quad k \in[2, N-1]_{\mathbb{Z}}, u_{k} \geq u^{2} .
$$

We will apply Theorem 2.3 with $g_{0}\left(k, u_{k}\right)=f\left(k, u_{k}\right)-r_{k} u_{k}$ and

$$
d_{k}=-r_{k} u^{2}-a(N-2) r_{k}^{+}+\min _{u_{k} \in\left[u^{2}, u^{2}+a(N-2)\right]} f\left(k, u_{k}\right), \quad \forall k \in[2, N-1]_{\mathbb{Z}} .
$$

Notice that

$$
\begin{aligned}
-r_{k} u_{k} & =r_{k}^{-} u_{k}-r_{k}^{+} u_{k} \geq r_{k}^{-} u^{2}-r_{k}^{+}\left(u^{2}+a(N-2)\right) \\
& =-r_{k} u^{2}-a(N-2) r_{k}^{+}, \quad \forall\left(k, u_{k}\right) \in[2, N-1]_{\mathbb{Z}} \times\left[u^{2}, u^{2}+a(N-2)\right],
\end{aligned}
$$

implying that (2.18) holds. Next, we have

$$
\overline{\mathbf{d}} \geq-u^{2} \overline{\mathbf{r}}-a(N-2) \overline{\mathbf{r}^{+}}+(\overline{\mathbf{r}}+\varepsilon) u^{2} \geq 0 .
$$

Hence, from Theorem 2.3 we deduce that (3.1) has an upper solution $\beta$ such that $u^{2} \leq \beta<$ $u^{1}+a(N-2)$. In particular $\alpha \leq \beta$, and, using Lemma 2.2, we infer that (3.1) has at least one solution $\mathbf{u}$ such that $\alpha \leq \mathbf{u} \leq \beta$, which is also a nontrivial solution.

Corollary 3.2 If $\mathbf{r}>0$ and

$$
\lim _{u \rightarrow 0_{+}} \frac{f(k, u)}{u}=0, \quad \lim _{u \rightarrow+\infty} \frac{f(k, u)}{u}=\infty, \quad \text { uniformly for } k \in[2, N-1]_{\mathbb{Z}},
$$

then (3.1) has at least one nontrivial solution.

Example 3.3 Consider the discrete Neumann problem with attractive singularity

$$
\nabla\left(\frac{\Delta u_{k}}{\sqrt{1-\kappa\left(\Delta u_{k}\right)^{2}}}\right)+r_{k} u_{k}=\left|u_{k}\right|^{\lambda}, \quad k \in[2, N-1]_{\mathbb{Z}}, \quad \Delta u_{1}=0=\Delta u_{N-1},
$$

where $\kappa>0$ is a constant, $\mathbf{r}=\left(r_{2}, \ldots, r_{N-1}\right) \in \mathbb{R}^{N-2}$ and $\lambda>0$. If $\mathbf{r}>0$ and $\lambda>1$, then the above problem has at least one solution. 


\subsection{Nonlinearities null at infinity}

In this section, we deal with nonlinearities null at infinity. This type of nonlinearities has been introduced in $[18]$ and studied in $[19,20]$. We consider the discrete Neumann problem

$$
\nabla\left[\phi\left(\Delta u_{k}\right)\right]+f\left(k, u_{k}\right)=s+\tilde{e}_{k}, \quad k \in[2, N-1]_{\mathbb{Z}}, \quad \Delta u_{1}=0=\Delta u_{N-1},
$$

where $f:[2, N-1]_{\mathbb{Z}} \times \mathbb{R} \rightarrow \mathbb{R}$ is a continuous function, $\tilde{\mathbf{e}}=\left(\tilde{e}_{2}, \ldots, \tilde{e}_{N-1}\right) \in \mathbb{R}^{N-2}$ with $\sum_{k=2}^{N-1} \tilde{e}_{k}=0$ and $s \in \mathbb{R}$ is a parameter. We have the following theorem.

\section{Theorem 3.4 Assume that}

$$
f(k, u) \rightarrow 0 \quad \text { if }|u| \rightarrow \infty \text { uniformly with } k \in[2, N-1]_{\mathbb{Z}},
$$

and there exists $\boldsymbol{v}=\left(v_{2}, \ldots, v_{N-1}\right) \in \mathbb{R}^{N-2}$ with $\overline{\boldsymbol{v}}=\frac{1}{N-2} \sum_{k=2}^{N-1} v_{k}>0$ such that

$$
\liminf _{|u| \rightarrow \infty} u f(k, u)>v \quad \text { uniformly with } k \in[2, N-1]_{\mathbb{Z}} .
$$

Then there exist $\varepsilon_{1}<0<\varepsilon_{2}$ such that (3.6) has no solutions if $s \notin\left[\varepsilon_{1}, \varepsilon_{2}\right]$ and at least one solution if $s \in\left[\varepsilon_{1}, \varepsilon_{2}\right]$. Moreover, if $s \in\left(\varepsilon_{1}, \varepsilon_{2}\right)$ and $s \neq 0$, then (3.6) has at least two solutions.

Proof For any fixed integer $n \in \mathbb{Z}$, let us consider the discrete Neumann problem

$$
\begin{aligned}
& \nabla\left[\phi\left(\Delta \tilde{u}_{k}\right)\right]+f\left(k, n+\tilde{u}_{k}\right)-\tilde{e}_{k}=\frac{1}{N-2} \sum_{j=2}^{N-1} f\left(j, n+\tilde{u}_{j}\right), \quad k \in[2, N-1]_{\mathbb{Z}}, \\
& \Delta u_{1}=0=\Delta u_{N-1} .
\end{aligned}
$$

Then, taking into account that $\sum_{k=2}^{N-1} \tilde{e}_{k}=0$, it follows from Lemma 2.3 that (3.9) has at least one solution, $\tilde{\mathbf{u}}^{(n)}=\left(\tilde{u}_{1}^{(n)}, \tilde{u}_{2}^{(n)}, \ldots, \tilde{u}_{N-1}^{(n)}, \tilde{u}_{N}^{(n)}\right) \in \tilde{W}^{N-2}$. Notice that $\mathbf{u}^{(n)}:=n+\tilde{\mathbf{u}}^{(n)}$ is a solution of (3.6) for $s=\frac{1}{N-2} \sum_{j=2}^{N-1} f\left(j, n+\tilde{u}_{j}^{(n)}\right)$. So, in particular, there exists at least one $s \in \mathbb{R}$ such that (3.6) has at least one solution.

Next, let us define

$$
S_{j}=\{s \in \mathbb{R} \mid(3.6) \text { has at least } j \text { solutions }\} \quad(j=1,2)
$$

and $\varepsilon_{1}=\inf S_{1}, \varepsilon_{2}=\sup S_{1}$. Using that $f$ is bounded on $[2, N-1]_{\mathbb{Z}} \times \mathbb{R}$ and $\frac{1}{N-2} \sum_{j=2}^{N-1} f\left(j, u_{j}\right)=$ $s$ for any solution $\mathbf{u}$ of (3.6), we infer that $\varepsilon_{1}, \varepsilon_{2}$ are finite.

Now, we will prove that $\varepsilon_{1}<0<\varepsilon_{2}$. It suffices to prove that there exists $\delta>0$ such that $[-\delta, \delta] \subset S_{1}$. One has

$$
\exists n_{0} \geq 1, \forall s \leq \frac{\bar{v}}{4 n_{0}}: \quad \frac{1}{N-2} \sum_{j=2}^{N-1} f\left(j, u_{j}^{\left(n_{0}\right)}\right) \geq s .
$$

Suppose on the contrary that

$$
\forall n \geq 1, \exists s_{n} \leq \frac{\bar{v}}{4 n}: \quad \frac{1}{N-2} \sum_{j=2}^{N-1} f\left(j, u_{j}^{(n)}\right)<s_{n} .
$$


Using (3.7), (3.8), and the fact that $\left\|\tilde{\mathbf{u}}^{(n)}\right\|_{\infty}<a(N-2)$ for all $n \in \mathbb{Z}$, it follows that there exists $X \geq 1$ such that

$$
\frac{1}{N-2} \sum_{j=2}^{N-1} f\left(j, u_{j}^{(n)}\right) u_{j}^{(n)} \geq \bar{v}
$$

and

$$
\frac{1}{N-2} \sum_{j=2}^{N-1} f\left(j, u_{j}^{(n)}\right) \tilde{u}_{j}^{(n)} \leq \frac{\bar{v}}{4}
$$

for all $n \geq X$. It follows that

$$
\begin{aligned}
0 & >\frac{n}{N-2} \sum_{j=2}^{N-1} f\left(j, u_{j}^{(n)}\right)-n s_{n} \\
& =\frac{1}{N-2} \sum_{j=2}^{N-1} f\left(j, u_{j}^{(n)}\right) u_{j}^{(n)}-\frac{1}{N-2} \sum_{j=2}^{N-1} f\left(j, u_{j}^{(n)}\right) \tilde{u}_{j}^{(n)}-n s_{n} \\
& \geq \frac{\bar{v}}{2}, \quad \text { for all } n \geq X
\end{aligned}
$$

which is a contradiction with the assumption $\bar{v}>0$. So, (3.10) holds true. This implies that $\mathbf{u}^{\left(n_{0}\right)}$ is a lower solution of (3.6) for all $s \leq \frac{\bar{v}}{4 n_{0}}$. Analogously, it follows that there exists $n_{1} \leq-1$ such that $\mathbf{u}^{\left(n_{1}\right)}$ is an upper solution of (3.6) for all $s \geq \frac{\bar{v}}{4 n_{1}}$. Then $[-\delta, \delta] \subset S_{1}$, just taking $\delta$ sufficiently small and applying Theorem 4 and Remark 8 of [14].

Next, let us prove that $\left(0, \varepsilon_{2}\right) \subset S_{2}$. Consider $s \in\left(0, \varepsilon_{2}\right)$. It follows that there exists $\hat{s}>s$ such that $\hat{s} \in S_{1}$, so, (3.6) has at least one solution $\alpha$ for $s=\hat{s}$. Then $\alpha$ is a strict lower solution of (3.6). Using once again (3.7) and the fact that $\left\|\tilde{\mathbf{u}}^{(n)}\right\|_{\infty}<a(N-2)$ for all $n \in \mathbb{Z}$, it follows that there exists $n \geq 1$ sufficiently large such that $\mathbf{u}^{(-n)}<\alpha<\mathbf{u}^{(n)}$ and

$$
\frac{1}{N-2} \sum_{j=2}^{N-1} f\left(j, u_{j}^{(l)}\right)<s \quad(l=-n, n) .
$$

It follows that $\mathbf{u}^{(-n)}, \mathbf{u}^{(n)}$ are strict upper solution for (3.6). Then from Lemma 2.2 we infer that (3.6) has a solution $\mathbf{v}^{1}$ such that $\alpha<\mathbf{v}^{1}<\mathbf{u}^{(n)}$. On the other hand, from Theorem 2.1, it follows that (3.6) has a solution $\mathbf{v}^{2}$ such that $u_{k}^{(-n)} \leq v_{k}^{2} \leq \alpha_{k}$ for some $k \in[1, N]_{\mathbb{Z}}$. Hence, $\mathbf{v}^{1} \neq \mathbf{v}^{2}$ and $s \in S_{1}$. Consider a sequence $s_{n}$ in $\left(0, \varepsilon_{2}\right)$ converging to $\varepsilon_{2}$ and $\mathbf{u}^{(n)}$ a solution of (3.6) with $s=s_{n}$. Notice that

$$
\frac{1}{N-2} \sum_{j=2}^{N-1} f\left(j, u_{j}^{(n)}\right)=s_{n} \quad(n \in \mathbb{N})
$$

which, together with $\left\|\tilde{\mathbf{u}}^{(n)}\right\|_{\infty}<a(N-2)$ for all $n \in \mathbb{N}, \varepsilon_{2}>0$ and (3.7), implies that $\left\{\overline{\mathbf{u}}^{(n)}\right\}$ is a bounded sequence. Consequently, $\left\{\mathbf{u}^{(n)}\right\}$ is a bounded sequence in $W^{N-2}$. Subsequently, there exists a subsequence of $\left\{\mathbf{u}^{(n)}\right\}$ converging uniformly to some $\mathbf{u} \in W^{N-2}$ which is a solution of (3.6) with $s=\varepsilon_{2}$. Analogously, one has $\varepsilon_{1} \in S_{1}$. 
Example 3.4 Consider the discrete Neumann problem

$$
\begin{aligned}
& \nabla\left(\frac{\Delta u_{k}}{\sqrt{1-\kappa\left(\Delta u_{k}\right)^{2}}}\right)+\frac{u_{k}}{1+\left(u_{k}\right)^{2}}=e_{k}+s, \quad k \in[2, N-1]_{\mathbb{Z}}, \\
& \Delta u_{1}=0=\Delta u_{N-1},
\end{aligned}
$$

where $\kappa>0$ is a constant, $\mathbf{e}=\left(e_{2}, \ldots, e_{N-1}\right) \in \mathbb{R}^{N-2}$ and $s \in \mathbb{R}$ is a parameter. From Theorem 3.4, there exist $\varepsilon_{1}<0<\varepsilon_{2}$ such that (3.11) has no solutions if $s \notin\left[\varepsilon_{1}, \varepsilon_{2}\right]$ and at least one solution if $s \in\left[\varepsilon_{1}, \varepsilon_{2}\right]$. Moreover, if $s \in\left(\varepsilon_{1}, \varepsilon_{2}\right)$ and $s \neq 0$, then (3.11) has at least two solutions.

Remark 3.1 It is interesting to note that in [14], the authors deal with nonlinearities $f \rightarrow$ $\infty$ at infinity for the discrete periodic problem, see [14, Theorem 6 and Theorem 7], which also hold for the discrete Neumann problem ([14, Remark 9]).

\subsection{Singular perturbations problem}

In the following we will apply Theorem 3.1 to study the singular Neumann problem

$$
\nabla\left[\phi\left(\Delta u_{k}\right)\right]+r_{k} u_{k}-\frac{m_{k}}{\left(u_{k}\right)^{\lambda}}=e_{k}, \quad k \in[2, N-1]_{\mathbb{Z}}, \quad \Delta u_{1}=0=\Delta u_{N-1},
$$

where $\mathbf{r}=\left(r_{2}, \ldots, r_{N-1}\right), \mathbf{m}=\left(m_{2}, \ldots, m_{N-1}\right), \mathbf{e}=\left(e_{2}, \ldots, e_{N-1}\right) \in \mathbb{R}^{N-2}$ and $\lambda>0$.

Theorem 3.5 Assume that $\overline{\mathbf{r}}>0, \mathbf{m} \geq 0$ with $\mathbf{m} \neq 0$ and

$$
\overline{\mathbf{e}}>a(N-2) \overline{\mathbf{r}^{+}}-\overline{\mathbf{m}}[a(N-2)]^{-\lambda}
$$

Then (3.12) has at least one positive solution.

Proof Let us define the auxiliary increasing functions

$$
\Psi_{1}(u)=a(N-2) \overline{\mathbf{r}^{+}}-\frac{\overline{\mathbf{m}}}{u^{\lambda}}, \quad \Psi_{2}(u)=u \overline{\mathbf{r}^{+}}-\frac{\overline{\mathbf{m}}}{u^{\lambda}}, \quad u>0 .
$$

From (3.13) it follows that $\overline{\mathbf{e}}>\Psi_{2}(a(N-2))$, and there exists $\varepsilon>0$ such that

$$
\overline{\mathbf{e}}>\Psi_{2}(a(N-2)+\varepsilon) .
$$

Now, consider the continuous function $g:[2, N-1]_{\mathbb{Z}} \times \mathbb{R} \rightarrow \mathbb{R}$ given by

$$
g(k, u)= \begin{cases}\frac{m_{k}}{u^{\lambda}}, & (k, u) \in[2, N-1]_{\mathbb{Z}} \times[\varepsilon, \infty), \\ \frac{m_{k}}{\varepsilon^{\lambda}}, & (k, u) \in[2, N-1]_{\mathbb{Z}} \times(-\infty, \varepsilon),\end{cases}
$$

and consider the modified Neumann problem

$$
\nabla\left[\phi\left(\Delta u_{k}\right)\right]+r_{k} u_{k}-g\left(k, u_{k}\right)=e_{k}, \quad \Delta u_{1}=0=\Delta u_{N-1} .
$$

Using that $g$ is bounded and $\overline{\mathbf{r}} \neq 0$, it follows from Theorem 3.1 that (3.15) has a solution $\mathbf{u} \neq 0$. 
We will show that $\min _{k \in[1, N]_{\mathbb{Z}}} u_{k}>\varepsilon$. Summing (3.15) from $k=2$ to $k=N-1$ we deduce that

$$
E=\sum_{k=2}^{N-1} r_{k}^{+} u_{k}-\sum_{k=2}^{N-1} r_{k}^{-} u_{k}-\sum_{k=2}^{N-1} g\left(k, u_{k}\right)
$$

which, together with Lemma 2.4, implies that

$$
\overline{\mathbf{e}} \leq a(N-2) \overline{\mathbf{r}^{+}}+\overline{\mathbf{r}} \min _{k \in[1, N]_{\mathbb{Z}}} u_{k}-\frac{1}{N-2} \sum_{k=2}^{N-1} g\left(k, u_{k}\right) .
$$

On the other hand, using $\mathbf{m} \geq 0$, one has

$$
\Psi_{2}(a(N-2)+\varepsilon) \geq a(N-2) \overline{\mathbf{r}^{+}}+\varepsilon \overline{\mathbf{r}}-\frac{\overline{\mathbf{m}}}{\varepsilon^{\lambda}} .
$$

Let us assume that $\max _{k \in[1, N]_{\mathbb{Z}}} u_{k} \leq \varepsilon$. Then, using (3.17) and $\overline{\mathbf{r}}>0$, we infer that

$$
\overline{\mathbf{e}} \leq a(N-2) \overline{\mathbf{r}^{+}}+\varepsilon \overline{\mathbf{r}}-\frac{\overline{\mathbf{m}}}{\varepsilon^{\lambda}},
$$

contradicting (3.14) and (3.18). So, $\max _{k \in[1, N]_{\mathbb{Z}}} u_{k}>\varepsilon$.

Next, using (3.17), (3.13), Lemma 2.4, and $\mathbf{m} \geq 0$, it follows that

$$
\begin{aligned}
0 & \leq \Psi_{1}\left(\max _{k \in[1, N]_{\mathbb{Z}}} u_{k}\right)-\overline{\mathbf{e}}+\overline{\mathbf{r}} \min _{k \in[1, N]_{\mathbb{Z}}} u_{k} \\
& <\Psi_{1}\left(\max _{k \in[1, N]_{\mathbb{Z}}} u_{k}\right)-\Psi_{1}(a(N-2))+\overline{\mathbf{r}} \min _{k \in[1, N]_{\mathbb{Z}}} u_{k} \\
& <\Psi_{1}\left(\min _{k \in[1, N]_{\mathbb{Z}}} u_{k}+a(N-2)\right)-\Psi_{1}(a(N-2))+\overline{\mathbf{r}} \min _{k \in[1, N]_{\mathbb{Z}}} u_{k},
\end{aligned}
$$

which, together with $\overline{\mathbf{r}}>0$, implies that $\min _{k \in[1, N]_{\mathbb{Z}}} u_{k}>0$.

From this, together with (3.14) and (3.16), we deduce that

$$
\Psi_{2}(a(N-2)+\varepsilon)<\overline{\mathbf{e}} \leq \Psi_{2}\left(\max _{k \in[1, N]_{\mathbb{Z}}} u_{k}\right),
$$

implying that $a(N-2)+\varepsilon<\max _{k \in[1, N]_{\mathbb{Z}}} u_{k}$. This, together with Lemma 2.4, implies that $\min _{k \in[1, N]_{\mathbb{Z}}} u_{k}>\varepsilon$, and our claim is proved. Consequently, $\mathbf{u}$ is also a solution of (3.12).

Remark 3.2 It is not difficult to show that the results proved in this paper also hold for the discrete periodic boundary value problem.

Example 3.5 Consider the discrete Neumann problem with attractive singularity

$$
\nabla\left(\frac{\Delta u_{k}}{\sqrt{1-\kappa\left(\Delta u_{k}\right)^{2}}}\right)+b u_{k}=\frac{1}{u^{\lambda}}, \quad k \in[2, N-1]_{\mathbb{Z}}, \quad \Delta u_{1}=0=\Delta u_{N-1},
$$

where $\kappa>0$ is a constant, $b>0$ and $\lambda \in(0,1)$. By using Theorem 3.5, the above problem has at least one solution if $b<\left(\frac{\sqrt{\kappa}}{N-2}\right)^{1+\lambda}$. 


\section{Competing interests}

The authors declare that there is no conflict of interests regarding the publication of this paper.

\section{Authors' contributions}

$Y L$ and RM completed the main study, carried out the results of this article and drafted the manuscript, YL checked the proofs and verified the calculation. All the authors read and approved the manuscript.

\section{Acknowledgements}

Research was supported by the NSFC (No. 11361054, No. 11201378), SRFDP (No. 20126203110004), Gansu provincial National Fundamental Research Science Foundation of China (No. 1208RJZA258).

\section{Received: 19 April 2014 Accepted: 30 July 2014 Published: 15 Aug 2014}

\section{References}

1. Calabi, E: Examples of Bernstein problems for some nonlinear equations. Proc. Symp. Pure Math. 15, $223-230$ (1970)

2. Cheng, SY, Yau, ST: Maximal spacelike hypersurfaces in the Lorentz-Minkowski spaces. Ann. Math. 104(2), 407-419 (1976)

3. Treibergs, A: Entire spacelike hypersurfaces of constant mean curvature in Minkowski space. Invent. Math. 66, 39-56 (1982)

4. López, R: Stationary surfaces in Lorentz-Minkowski space. Proc. R. Soc. Edinb. 138, 1067-1096 (2008)

5. Bereanu, C, Mawhin, J: Existence and multiplicity results for some nonlinear problems with singular $\phi$-Laplacian. J. Differ. Equ. 243, 536-557 (2007)

6. Bereanu, C, Jebelean, P, Mawhin, J: Radial solutions for Neumann problems with $\phi$-Laplacians and pendulum-like nonlinearities. Discrete Contin. Dyn. Syst. 28(2), 637-648 (2010)

7. Bereanu, C, Jebelean, P, Mawhin, J: Radial solutions for Neumann problems involving mean curvature operators in Euclidean and Minkowski spaces. Math. Nachr. 283(3), 379-391 (2010)

8. Sun, JP, Li, WT: Multiple positive solutions to second-order Neumann boundary value problems. Appl. Math. Comput. 146, 187-194 (2003)

9. Chu, J, Sun, Y, Chen, H: Positive solutions of Neumann problems with singularities. J. Math. Anal. Appl. 337(2), 1267-1272 (2008)

10. Cabada, A, Habets, P, Pouso, RL: Optimal existence conditions for $\phi$-Laplacian equations with upper and lower solutions in the reversed order. J. Differ. Equ. 166, 385-401 (2000)

11. Hooker, JW: Some differences between difference equations and differential equations. J. Differ. Equ. Appl. 2, 219-225 (1996)

12. Kelley, WG, Peterson, AC: Difference Equations: An Introduction with Applications. Academic Press, New York (1991)

13. Bereanu, C, Thompson, HB: Periodic solutions of second order nonlinear difference equations with discrete $\phi$-Laplacian. J. Math. Anal. Appl. 330, 1002-1015 (2007)

14. Bereanu, C, Mawhin, J: Boundary value problems for second-order nonlinear difference equations with discrete $\phi$-Laplacian and singular $\phi$. J. Differ. Equ. Appl. 14(10-11), 1099-1118 (2008)

15. Cabada, A, Otero-Espinar, V: Fixed sign solutions of second-order difference equations with Neumann boundary conditions. Comput. Math. Appl. 45, 1125-1136 (2003)

16. Anderson, DR, Rachunková, I, Tisdell, CC: Solvability of discrete Neumann boundary value problems. J. Math. Anal. Appl. 331, 736-741 (2007)

17. Bereanu, C, Gheorghe, D, Zamora, M: Periodic solutions for singular perturbations of the singular $\phi$-Laplacian operator. Commun. Contemp. Math. 15(4), 1250063 (2013)

18. Fučik, S: Further remark on a theorem by E. M. Landesman and A. C. Lazer. Comment. Math. Univ. Carol. 15, $259-271$ (1974)

19. Bereanu, C, Mawhin, J: Multiple periodic solutions of ordinary differential equations with bounded nonlinearities and $\phi$-Laplacian. NoDEA Nonlinear Differ. Equ. Appl. 15, 159-168 (2008)

20. Bereanu, C, Gheorghe, D, Zamora, M: Non-resonant boundary value problems with singular $\boldsymbol{\phi}$-Laplacian operators. NoDEA Nonlinear Differ. Equ. Appl. 20, 1365-1377 (2013)

10.1186/1687-1847-2014-227

Cite this article as: Lu and Ma: Existence and multiplicity of solutions of second-order discrete Neumann problem with singular $\phi$-Laplacian operator. Advances in Difference Equations 2014, 2014:227 